\title{
La formación inicial y continua de los docentes en los países del MERCOSUR. Problemas comunes, estructuras y desarrollos diversos
}

\author{
Initial and continuous teacher training in \\ MERCOSUR member States. Common issues, \\ different structures and developments
}

\begin{abstract}
Andrea Alliaud
Doctora en Educación por la Universidad de Buenos Aires (UBA). Docente-investigadora de la Facultad de Filosofía y Letras (UBA). Profesora de Posgrado en la Universidad Torcuato Di Tella. Coordinadora del Área de Formación Docente en la OEl de Argentina. Especialista en Formación Docente.

Lea Vezub

Doctora en Educación por la Universidad de Buenos Aires. Docente e Investigadora de la Facultad de Filosofía y Letras, Universidad de Buenos Aires. Profesora Adjunta del Dpto. de Humanidades de la Universidad Nacional de Moreno. Consultora internacional, especialista en formación docente y metodología de la investigación educativa.
\end{abstract}

Fecha de recibido: 14/02/2014

Fecha de aceptación: 11/06/2014

\section{Resumen}

A pesar de haber sido objeto de cambios y transformaciones recientes, las estructuras, planes y normas que organizan los sistemas de formación docente inicial y continua en los países del Mercado Común del Sur (MERCOSUR) siguen en debate. En este contexto se plantea el desafío de encontrar puntos de articulación que contribuyan a la integración regional de los países del bloque y a la movilidad de los docentes.

Si bien la currícula y los programas de formación continua constituyen sólo una parte o dimensión del complejo proceso de la formación docente, su análisis y comparación resultan relevantes y oportunos toda vez que se pretende mejorar la preparación de los maestros y profesores. En este marco cabe preguntarse cuáles son las principales tendencias que caracterizan a las propuestas de formación en la región.

El artículo resume los principales resultados alcanzados a través de un estudio llevado a cabo a solicitud del Programa de Apoyo al Sector Educativo del Mercosur (PASEM) cuyo propósito fue comparar los planes de formación docente inicial así como la oferta y organización de la formación continua existente en los siguientes países miembro-fundadores del MERCOSUR: Argentina, Brasil, Uruguay y Paraguay.

La metodología seguida se focalizó en el relevamiento y análisis comparado de los documentos, lineamientos y programas de formación inicial y continua. Se trabajó en base a una serie de problemas, dimensiones y categorías de análisis con el objetivo de destacar aspectos compatibles y otros singulares que fueran significativos tanto a los fines de la integración como al mejoramiento de la formación docente.

PALABRAS CLAVE: formación - docente - programa de formación de docentes perfeccionamiento 


\section{Abstract}

Despite changes and recent transformations, the organizing structures, plans and rules of initial and continuous teacher training in countries of MERCOSUR (Common Market of the South) are still under debate. Within this context, the challenge posed is to find points of articulation that would contribute to a better regional integration of the member States of the MERCOSUR as well as to the teachers' mobility.

Even though the plans of studies and programs for continuing teacher training are just a part or an area of a larger and complex process of teacher training, studying and comparing those plans and programs become most relevant every time the improvement of teachers' training is intended. Within this framework, it becomes relevant to discuss the main trends that describe the teacher training proposals in the region.

The article summarizes the main results that arise from a research carried out upon request of the "Programa de Apoyo al Sector Educativo del MERCOSUR" (PASEM -Support Program for Education Sector of MERCOSUR). The purpose of said research was to compare the plans of initial teacher training and the proposals and structure of existing initiatives for continuing teacher training among the founding member states of MERCOSUR, that is Argentina, Brazil, Uruguay and Paraguay.

The methodology applied focused on the survey and comparative analysis of documents, guidelines and programs of initial and continuing teacher training. The research was based on a wide range of issues, dimensions and categories of analysis with the purpose of emphasizing those aspects in common as well as particular ones that were significant both for the integration purpose and for the improvement of teacher training in the whole region.

KEYWORDS: initial teacher training- teacher training programs- teacher professional development

\section{Introducción}

Este artículo resume los principales resultados alcanzados a través de un estudio llevado a cabo en el marco del PASEM ${ }^{1}$, cuyo propósito fue comparar los diferentes planes de formación docente inicial, la oferta y organización de la formación continua existente en los siguientes países miembro-fundadores del MERCOSUR (Argentina, Brasil, Uruguay y Paraguay) 2.

Entre los objetivos estratégicos del Plan del Sector Educativo del MERCOSUR 2011-2015 se destacan los siguientes: "Contribuir a la integración regional acordando y ejecutando políticas educativas que promuevan una ciudadanía regional, una cultura de paz y el respeto a la democracia, a los derechos humanos y al medio ambiente"; "Promover la educación de calidad para todos como factor de inclusión social, de desarrollo humano y productivo"; "Impulsar y fortalecer programas de movilidad de estudiantes, pasantes, docentes, investigadores, gestores, directivos y profesionales".

En este marco, la formación y profesionalización de los docentes constituyen un punto estratégico en la agenda de las políticas públicas nacionales y de la región. Surgió así la demanda de análisis y comparación de los sistemas de formación docente de los países miembros con el objetivo de acordar políticas tendientes a fortalecer y articular los sistemas formadores como parte del proceso de integración del MERCOSUR que prevé, entre otros aspectos, la movilidad docente. El examen realizado sobre los planes y la oferta de formación continua representa un aporte en este sentido. A lo largo de su desarrollo se procuró identificar parámetros comunes o compatibles en la normativa vigente en el MERCOSUR y también rasgos propios de cada país, considerados relevantes para la mejora y articulación de los sistemas formadores. El estudio comparado se focalizó en los aspectos que merecen ser considerados, cuestionados, revisados y mejorados a fin de generar condiciones para la formulación de políticas docentes de integración regional con el propósito de avanzar hacia una convergencia, sin unificar a la fuerza los sistemas formadores propios de cada país. 


\section{Metodología y alcances del estudio}

Con la intención de obtener un informe analítico y no meramente descriptivo de las estructuras, organización y oferta de la formación docente inicial y continua, en primer lugar se consideraron las transformaciones recientes ocurridas en la formación y en los sistemas educativos contemporáneos. El primer desafío para avanzar en la comparación fue identificar y definir una serie de problemas actuales, de tensiones comunes para los cuatro países, frente a los que la formación inicial y en servicio se vea desafiada, interpelada. La discusión arrojó una serie de problemas a la luz de los cuales se describieron y compararon los respectivos sistemas formadores. Éstos quedaron formulados para la formación inicial de la siguiente manera (Cf. Alliaud, 2010; Davini, 2005; Terigi, 2012).

- Con frecuencia, la formación docente fue cuestionada debido a su dificultad para incorporar los temas y problemas "reales" de las escuelas para las que forma. Uno de los interrogantes se dirigió, por lo tanto, a indagar si los planes vigentes dialogan con los temas y problemas que exige el desempeño del oficio docente hoy (nuevas infancias y adolescencias, trasformaciones sociales y culturales, igualdad/desigualdad, multiculturalismo, etc.).

- Los cambios curriculares recientes han intentado superar la dicotomía o divorcio entre la teoría y la práctica en la formación. Sin embargo persisten dificultades visibles, sobre todo en los docentes recién formados quienes perciben frecuentemente que su preparación no es suficiente para intervenir en las situaciones reales de enseñanza. Es probable que los planes hayan avanzado poco en explicitar cuáles serían los dispositivos de formación más pertinentes ${ }^{3}$ para integrar instancias del saber y del hacer, por lo que se hace necesario poner especial atención sobre lo que se plantea al respecto.

- El abordaje de las prácticas formativas también ha sido fuertemente cuestionado tanto por sus concepciones subyacentes como por su lógica, forma de organización, secuenciación, etc. Muchas veces las prácticas profesionales se han realizado en ámbitos y contextos no representativos de las instituciones en las que luego se insertan los graduados. Por ello el análisis de los planes debe atender especialmente al lugar, contenido, secuencia y formas de organización de la práctica, así como a la relación y características de las escuelas que las instancias de formación incorporan.

- La docencia constituye además un trabajo, y como tal presenta una lógica asociada con la organización institucional del puesto laboral que no debería desestimarse durante la formación. ¿Cómo conciben los planes de estudio el trabajo docente? ¿Cómo forman para el trabajo individual o colectivo? ¿Incluyen en la formación temas referidos a su identidad, autonomía y responsabilidad profesional?

- Tradicionalmente los planes docentes que forman para el nivel secundario han separado la formación disciplinar de la pedagógica, otorgando a la primera un peso sustantivo en desmedro de la segunda. Resulta relevante analizar si los nuevos diseños mantienen o no dicha desvinculación ¿Qué peso y relevancia tienen uno y otro campo de formación en los planes? ¿Cómo se resuelve esta tensión histórica?

- La evaluación de los planes y los procesos de formación suele ser un tema ausente en varios de los países de la región, lo cual dificulta contar con información relevante para definir cambios y emprender procesos de mejora. En este sentido, resulta pertinente analizar si los diseños contemplan mecanismos de evaluación y seguimiento que permitan valorar los procesos de formación emprendidos.

Por su parte, la revisión de la bibliografía y el estado de la cuestión sobre las políticas de formación continua y el desarrollo profesional docente permiten identificar cuatro grandes problemas (Cf. Ávalos, 2007; Vaillant, 2005; Vezub, 2007; 2013).

- La gestión de las políticas. La formación continua evidencia una baja articulación entre los diferentes niveles de gobierno (nacionales, provinciales / estatales) que llevan adelante 
las acciones, o entre áreas y programas de un mismo ámbito o jurisdicción. También ha sido frecuente la interrupción de las políticas emprendidas entre distintas gestiones, así como la falta de evaluación sistemática de los resultados alcanzados a través de las actividades de formación en servicio.

- La regulación normativa. Frente a la ausencia de normativas y parámetros que orienten las propuestas, en la formación continua ha sido frecuente la aparición de lógicas de mercado y el predominio del credencialismo que impulsa a obtener puntaje para ascender en la carrera docente excluyendo otras motivaciones. La oferta masiva de cursos no siempre asegura su calidad y cómo será su contribución a las necesidades y prioridades del sistema educativo. Los programas no siempre responden a los problemas de la enseñanza y a la práctica docente situada. De este modo, la capacitación no ha logrado favorecer el desarrollo de las trayectorias profesionales docentes y el mejoramiento de las experiencias educativas de los alumnos.

- Oferta poco variada. La formación permanente se ha caracterizado por presentar una oferta homogénea y homogeneizadora de las prácticas de sus destinatarios. Los formatos y dispositivos de formación (cursos, talleres, seminarios) son poco variados y entre sus temáticas predominan las áreas curriculares básicas, con vacancia de ciertos contenidos del currículum, o de temas vinculados con determinadas especialidades, modalidades o contextos del sistema educativo. Por lo general, no hay programas de formación de formadores y la formación de roles y funciones está centrada casi exclusivamente en los cargos de gestión y supervisión de las instituciones, sin contemplar otras tareas docentes que actualmente requiere el sistema.

- Modelos y enfoques de formación. El énfasis en el saber experto y la desvinculación de las propuestas de formación continua respecto de los problemas de la práctica docente es otro de los aspectos críticos que la caracterizan. Con frecuencia, la propuesta formativa ha sido dirigida al docente individual, con menor incidencia en los equipos y colectivos institucionales. Dadas estas circunstancias, la formación continua presenta dificultades a la hora de modificar prácticas y mejorar la enseñanza y los entornos escolares.

En relación con los problemas enunciados, el estudio se propuso examinar en qué ha avanzado la región y cuáles son todavía las dificultades, con vistas a lograr posibles puntos de convergencia en el proceso de articulación regional. Como se verá, se observan avances sustantivos en ciertas áreas en algunos países, mientras que permanecen dificultades en otras problemáticas. La institucionalización reciente de la formación docente continua en relación con la formación inicial es un aspecto a considerar ya que supone partir de cierto retraso respecto de las políticas, las conceptualizaciones y los recursos con los que se cuenta. Así se entiende que en muchos casos los logros de los distintos países sean mayores y más consistentes en la esfera de la formación inicial que en la formación continua.

Una vez consensuados los problemas en torno a los cuales analizar los planes y la normativa que regula la formación en servicio, la segunda tarea fue identificar y circunscribir el corpus bajo análisis. En el caso de la formación inicial fue más sencillo, ya que se encuentra establecido en los planes o diseños curriculares de cada país y en los marcos legales que la regulan. Para la formación en servicio, en cambio, la identificación del corpus empírico tuvo más dificultades, dada la reciente institucionalización de la formación permanente (ya señalada) y la mayor amplitud, dinámica, flexibilidad y heterogeneidad que suele presentar respecto de la formación inicial. Asimismo, su grado de regulación suele ser mucho menor. La oferta de cursos, posgrados u otras actividades de desarrollo profesional se encuentra muchas veces dispersa entre las diversas instituciones que la imparten y, es a la vez bastante cambiante, por lo que el análisis se circunscribe muchas veces a la información disponible y capturada en el momento de su búsqueda.

El tercer desafío metodológico fue comparar países con estructuras de gobierno federales y con sistemas nacionales centralizados. En Uruguay y Paraguay la formación docente es nacional, lo que significa que todas las instituciones que la imparten se ajustan a los mismos 
planes de estudio y normas. Por el contrario, en Argentina y Brasil, al tratarse de países federales existen lineamientos o directrices nacionales de carácter general que coexisten con la autonomía de los gobiernos locales para la definición de políticas y diseños curriculares a nivel provincial (Argentina) o estatal (Brasil) y con la potestad de las propias Universidades que ofrecen carreras de formación docente. En los países federales se consideraron algunos estados o provincias como casos de análisis.

En cuanto a los métodos, se acudió principalmente a documentos e información estadística (fuentes primarias) y, de manera subsidiaria, a la consulta de informantes clave: responsables de gobierno y gestión de las áreas correspondientes. Estas fueron utilizadas para corroborar algunas interpretaciones sobre los documentos, informar acerca de los procesos de construcción curricular, así como de la organización y funcionamiento de la oferta de formación permanente.

\section{La Formación Docente Inicial en el MERCOSUR}

\section{El lugar del Estado y de la regulación curricular}

En los países estudiados la formación docente se desarrolla en el nivel superior: institutos o centros y universidades. En un extremo podría mencionarse el caso de Uruguay, país en que la formación se lleva a cabo principalmente en institutos superiores, ya que las universidades tienen un protagonismo menor y muy reciente ${ }^{4}$. En el otro extremo se ubica Brasil, donde la formación docente es impartida por las universidades aunque persistan todavía docentes que se forman en el nivel medio para desempeñarse en educación infantil y los años iniciales de la Enseñanza Fundamental. De todos modos, hay un fuerte debate para que la formación se imparta exclusivamente en el nivel superior. En un punto intermedio se encuentran Argentina y Paraguay, países en que los institutos superiores cobran protagonismo en la preparación de docentes, aunque se registra una importante oferta de universidades que dictan carreras, principalmente, para la formación de profesores de nivel secundario.

Tanto en Brasil como en Argentina y en Uruguay la formación docente que imparten las instituciones públicas es gratuita (como el resto de la educación superior); la excepción la constituye Paraguay donde la formación superior es arancelada.

En todos los países, si bien existen regulaciones del Estado, éstas varían en su alcance. En Uruguay los diseños curriculares y programas nacionales regulan toda la oferta de formación, tanto la del sector público como la del privado. En Brasil las directrices nacionales presentan un carácter general y las universidades tienen amplia autonomía para formular sus planes, aunque este control menor de las orientaciones iniciales se incrementa posteriormente a través de los mecanismos de evaluación y acreditación de las instituciones y sus ofertas. En el caso de Paraguay, los diseños nacionales alcanzan al ámbito privado, mientras que las universidades tienen autonomía para diseñar sus propios planes aunque afirman respetar algunas de las disposiciones establecidas por el Estado. En Argentina, la norma nacional regula la totalidad del sistema formador (público y privado) y plantea que las universidades se acojan a los mismos lineamientos. No obstante, recién en los últimos años las universidades nacionales han iniciado un proceso de adecuación curricular de sus carreras docentes al producirse su incorporación a la Ley Nacional de Educación Superior (art. 43) como carreras de "interés público".

Un rasgo común entre los países del MERCOSUR es el retorno al control que el Estado ejerce sobre la formación docente, lo que podría explicarse en virtud de la importancia otorgada a la docencia para el mejoramiento de la calidad de los sistemas educativos. La regulación que ejerce el Estado varía en función del tipo de instituciones que imparten la formación: en aquellos países donde las universidades cobran protagonismo (Brasil) el control estatal se da fundamentalmente a través de mecanismos de evaluación y acreditación; en cambio, cuando la oferta se concentra en institutos superiores (Argentina, Uruguay, Paraguay) el control estatal se manifiesta sobre todo en las instancias previas, a través de disposiciones más fuertes en los procesos de definición curricular. 


\section{La extensión y duración de la formación inicial}

La extensión y homologación de la duración del conjunto de las carreras de formación docente es una tendencia a nivel mundial que procura su jerarquización y articulación con ofertas formativas de posgrado. La consideración de la duración de las carreras que forman docentes constituye un aspecto clave a la hora de plantear criterios para la integración regional. Al contemplar la experiencia del Espacio Europeo de Educación Superior (EEES), uno de los puntos de convergencia a los que se llegó fue precisamente la duración de las carreras universitarias, entre las que se encuentran las docentes; el otro se refiere a un sistema de acreditación de estudios que permite la movilidad de estudiantes entre las distintas universidades de la comunidad.

En los países del MERCOSUR se advierte una semejanza estructural en cuanto a la duración de las carreras de formación docente que oscila entre los 3 y 4 años y 3.000 horas en promedio ${ }^{5}$. Existen sin embargo matices: en Argentina la duración y extensión es común para todas las carreras docentes, mientras que en el resto de los países la carga horaria aumenta levemente para el caso de los profesorados de nivel inicial y/o primario, en virtud de la mayor carga horaria de práctica estipulada para estos profesorados. Esta tendencia se opone a la imperante por muchos años según la cual la formación de docentes para los primeros niveles del sistema requería una formación menor en cantidad de años de estudio. Ante dicho cambio surge el interrogante acerca de la relación entre la nueva duración de las carreras y las escalas salariales: si éstas mantienen la diferenciación histórica (mayores salarios para docentes que trabajan en los niveles más altos del sistema), se produciría una extraña confluencia entre carreras más largas y menores salarios y viceversa.

Tal como se señaló, se observan en la región duraciones equiparables entre las carreras que forman docentes, considerando la formación de grado. La tendencia a articular la formación inicial con instancias de posgrado más especializadas y prolongadas se refleja sólo en Brasil que está trabajando en esa dirección.

\section{La flexibilidad curricular en los estudios de pregrado}

La factibilidad de avanzar en proyectos de integración regional y de movilidad de los docentes requiere atender los aspectos "más duros" de las normas (cantidad de horas, duración de los planes, etc.) y aquellos habitualmente entendidos como "más blandos", entre los cuales se destaca la flexibilidad curricular por su grado de pertinencia ${ }^{6}$.

El grado de apertura de los planes de formación docente de los distintos países de la región se observa, por ejemplo, en la habilitación de espacios curriculares que aborden problemáticas regionales, así como en la incorporación de otras lenguas (el español, el portugués, el guaraní, entre otros). Asimismo, es preciso examinar en qué medida los contenidos de la formación favorecen el desarrollo de capacidades, competencias o habilidades que permitan la "apertura" necesaria para comprender otras realidades y escenarios más allá de los locales.

En el caso de Argentina, el documento central prevé instancias, unidades curriculares, de definición institucional (hasta un $20 \%$ de la carga horaria del curriculum) que son de cursado opcional para los estudiantes y también se contemplan formas de acreditación de otras experiencias de formación a través de actividades "menos escolarizadas", tales como la asistencia a jornadas, conferencias, coloquios, ciclos de arte, debates, etc. También en ese país, al igual que en Uruguay y Paraguay, hay un espacio curricular destinado a una lengua extranjera (en Paraguay sólo se incluye en el plan de nivel primario). En Brasil, las universidades ofrecen -a través de sus centros de idiomas- lenguas extranjeras como actividades extra curriculares.

Los diseños para el nivel inicial y para el profesorado de la educación media de Paraguay prevén dos instancias optativas para los alumnos. Asimismo, las carreras de nivel medio 
enfatizan la participación de los estudiantes en la definición de su ruta de aprendizaje. Los diseños permiten la equiparación, convalidación u homologación entre programas de formación docente del país y del extranjero, y los contenidos contemplan su adecuación a las nuevas realidades económicas, políticas, sociales y legales del país, de la región y del mundo. En Brasil los planes de estudio de las universidades contienen unidades opcionales y los estudiantes pueden cursar asignaturas de su elección en otras instituciones. Asimismo establecen la posibilidad de acreditar actividades académico-científico-culturales.

El análisis efectuado muestra países que presentan mayor grado de apertura o planes de estudio de formación más abiertos y flexibles, como el de Brasil y Argentina; Paraguay, con menor apertura y Uruguay, con una estructura más cerrada aunque se trata de diseños posibles de ser equiparados a los de los otros países.

Por último, en los procesos de integración regional es importante atender a la evaluación de los planes de estudio: se trata de un aspecto también atinente al criterio de flexibilidad ya que permite la permanente revisión y ajuste de los planes a partir de acuerdos y prioridades regionales. Los lineamientos de Argentina conciben al curriculum de la formación desde una perspectiva dinámica; la evaluación curricular se define como una tarea y un proceso permanente que incluye un trabajo conjunto entre las instituciones y las autoridades provinciales. En Brasil la evaluación curricular de las universidades se realiza conforme a las normas del Ministerio de Educación, organismo que regula la autorización y el reconocimiento de los títulos. En Paraguay, la Dirección de Formación Docente cuenta con un Diseño de Evaluación del curriculum que se lleva adelante en diálogo con los institutos formadores; a partir de los resultados recogidos, los diseños curriculares se ajustan y se elaboran nuevas formulaciones para un posterior período de implementación. En el caso uruguayo, el Plan es sucinto al precisar los alcances y procedimientos de la evaluación curricular; sólo establece que la Dirección de Formación Docente realizará un seguimiento y evaluación del nuevo plan a efectos de su posible revisión a través de grupos de trabajo e informes anuales.

\section{Acerca de los perfiles e identidades docentes}

Dada la importancia estratégica asignada por las políticas educativas a los docentes y a su trabajo (complejizado y dificultado en las últimas décadas), se consideró relevante reparar en esta dimensión: cómo se lo concibe y qué tipo de docente se aspira formar. Los "perfiles docentes" están presentes en todos los planes de estudio e incluyen un conjunto de rasgos acerca de lo que cada país espera de la intervención / desempeño de sus profesores en las instituciones educativas para las que se forman. Por lo tanto, se trata de otro de los criterios a ser contemplado en procesos de integración regional.

En relación a esta categoría, los países del MERCOSUR han realizado distintas opciones: Argentina y Uruguay definen una serie de capacidades inherentes a la concepción del docente que se espera formar, en tanto que Brasil y Paraguay desarrollan el perfil en términos de competencias. Sin entrar en la discusión acerca de la adopción de un término u otro o de los enfoques teóricos que sustentan a cada uno, estas denominaciones muestran la preocupación de los países por la formación de docentes que "puedan hacer", es decir, que sean capaces de actuar, de tomar decisiones fundadas y de indagar sobre sus prácticas educativas.

Al analizar las capacidades o competencias propuestas en los currículos de formación inicial de los países, se evidencia que son relevantes para enfrentar los desafíos actuales $\mathrm{y}$, asimismo, que pueden compatibilizarse y complementarse. Argentina y Brasil conciben al docente como un trabajador de la cultura: en el caso del primero su desempeño se circunscribe fundamentalmente a los contextos escolares, mientras que en el segundo se enfatizan además los ámbitos "no escolares".

Algunos de los denominadores comunes que presentan los documentos curriculares estudiados son: la preocupación por la enseñanza; la reflexión y comprensión de las 
dimensiones sociopolíticas, histórico-culturales, pedagógicas, metodológicas y disciplinarias para un adecuado desempeño en las escuelas; la incorporación de los contextos sociales y las problemáticas contemporáneas; el compromiso con los valores democráticos; la comprensión del papel social de la escuela; el dominio de los contenidos, su significado en diferentes contextos y su articulación interdisciplinar; el conocimiento de los procesos de investigación; el desarrollo profesional y la formación permanente; la dimensión ética de la profesión.

Debido a lo anterior puede afirmarse que las políticas de integración regional podrían contemplar el conjunto de las capacidades / competencias propuestas por los planes de cada país y definir un perfil docente para la región que las abarque, a fin de formar un docente acorde con los desafíos que presenta la profesión en la actualidad. Este perfil podría fundarse en las capacidades comunes que surgen del análisis curricular realizado, las que se encuentran vinculadas a las dimensiones más relevantes para repensar la profesión en el siglo $\mathrm{XXI}$. Nos referimos a las pedagógico-didácticas, interactivas / comunicativas, institucionales, sociopolíticas, contextuales / situacionales y productivas ${ }^{7}$.

\section{El lugar de la práctica pre-profesional}

La práctica pre-profesional ha cobrado protagonismo en los planes de formación, no sólo debido a su acrecentamiento sino también por su tratamiento diferente. Se han superado las tradiciones deductivas que la colocaban al final de los planes de estudio para incorporarlas desde los inicios de la formación, contemplando distintas dimensiones del quehacer profesional (institucional, del aula, de la comunidad, etc.). Su abordaje situado y contextualizado permite formar docentes que puedan desempeñarse en contextos y escenarios educativos reales.

En los países del MERCOSUR, la formación práctica se lleva a cabo desde los primeros años. No obstante, en varias universidades argentinas ${ }^{8}$ y en la mayoría de las paraguayas los planes de estudio mantienen una estructura más tradicional. En los casos de Brasil, Paraguay y Uruguay, como se ha señalado, la carga horaria prevista para las prácticas es mayor para las carreras de educación infantil y primaria que para las de secundaria. En Argentina hay un porcentaje de prácticas que deben respetar todos los planes de formación docente.

Si bien las prácticas se desarrollan desde el principio de la formación, no significa que los estudiantes comiencen a dar clases en los primeros años de la carrera. La aproximación a los espacios de la práctica profesional suele ser progresiva y gradual en los trayectos formativos de los planes estudiados. Las normativas y los diseños curriculares de la región incorporan un enfoque situado y contextualizado de las prácticas docentes, alertando sobre la importancia de efectivizarlas en escenarios y tipos de instituciones variados.

Aunque se expresa la necesidad de articular los espacios de la práctica con otros campos de la formación ${ }^{9}$, la asociación de las instancias de formación teórica con los espacios de la práctica ha sido y sigue siendo un problema difícil de superar en las carreras docentes: las prácticas suelen concebirse como ámbitos destinados al "hacer", mientras que los otros campos de la formación se estructuran a partir de conocimientos formalizados, casi exclusivamente destinados al "saber". Al respecto parece novedosa y superadora de esta problemática la propuesta de Brasil, que concibe la práctica como un componente curricular de los distintos espacios formativos. En esta propuesta las prácticas profesionales atraviesan la totalidad del curriculum, antes que presentarse como un espacio aislado que requiere posteriores esfuerzos de articulación con el resto. En los planes de profesorado para el nivel secundario de Uruguay las prácticas forman parte de las didácticas específicas, sin vinculación con el resto del plan, como en el caso de Brasil.

El análisis realizado permite apreciar que en los países del MERCOSUR prevalecen los "modelos concurrentes" en todos los planes de profesorado, es decir, las prácticas se desarrollan de manera simultánea a los demás espacios curriculares. El tratamiento de las 
prácticas (con todos sus componentes) tiene gran relevancia en las normativas nacionales y es una prescripción que se encuentra regulada en los planes de formación docente de los países de la región.

\section{La Formación Docente Continua en el MERCOSUR}

\section{La oferta y regulación de la formación en servicio}

En Argentina, Paraguay y Uruguay el Estado organiza la formación docente continua. El Ministerio de Educación de Argentina proporciona orientaciones generales y ofrece algunas acciones de manera directa a través de Postítulos o Programas Nacionales, pero son las provincias las que elaboran y ejecutan la mayoría de las propuestas de formación continua a través de sus propios organismos e instituciones educativas oficiales, gremios o universidades. Es decir que se combina una oferta centralizada con actividades descentralizadas, implementadas por los gobiernos locales pero bajo lineamientos u objetivos estratégicos nacionales.

En Paraguay las acciones de formación continua son impulsadas y ofertadas por el Ministerio de Educación y Cultura a nivel central, con apoyo del Instituto Superior de Educación Dr. Raúl Peña de Asunción y a través del Sistema Nacional de Actualización Docente. Uruguay también cuenta con una política centralizada que canaliza el Instituto de Perfeccionamiento y Estudios Superiores de Montevideo mediante cursos y posgrados, a los que se agregan programas nacionales específicos que tienen su propio componente de capacitación.

Brasil es de los cuatro países el que presenta menor nivel de regulación de la oferta de capacitación docente: no posee una ley o directriz específica. El Estado Federal actúa fundamentalmente a través de los fondos de financiamiento concursables y sólo coordina las políticas ya que las acciones de formación continua dependen de la articulación y colaboración que se generen entre las instituciones formadoras y el sistema educativo. La formación continua se organiza a través de: (i) las universidades (públicas y privadas) mediante cursos de extensión y posgrados; (ii) el Ministerio de Educación a través de la Coordinación de Perfeccionamiento de Personal de Nivel Superior (CAPES) que articula los tres niveles de gobierno y las universidades públicas federales y estatales y; (iii) las secretarías de educación estatales y municipales.

Argentina es el único de los cuatro países del MERCOSUR que formuló un documento específico titulado Lineamientos Nacionales para la Formación Docente Continua y el Desarrollo Profesional, donde se proponen criterios para orientar las acciones de desarrollo profesional nacionales, provinciales e institucionales. Asimismo, una nueva Resolución creó el Programa Nacional de Formación Permanente que contempla los diversos niveles y sectores del sistema y se propone articular procesos de formación con mecanismos de evaluación y fortalecimiento de la escuela. Este programa posee un componente centrado en las instituciones educativas con dos recorridos según la función desempeñada (directores / supervisores versus equipos docentes); y un segundo componente para destinatarios específicos que atiende a las trayectorias, niveles, especialidades, roles y temas priorizados por cada jurisdicción.

En Uruguay las normas que rigen la formación continua se refieren a aspectos formales de los cursos (duración, titulaciones, modalidades, evaluación y emisión de certificados). En los últimos años se reconoció el derecho de los docentes a realizar estudios de posgrado. A tal fin se elaboró una oferta específica y se estableció un reglamento para los posgrados dictados por el IPES en convenio con la Universidad de la República (UdelaR).

En Paraguay tampoco hay una normativa nacional con criterios generales, sino recomendaciones sobre las modalidades y los temas de formación para los que se elaboran módulos de contenidos mínimos. El Ministerio establece una serie de "profesionalizaciones"10 que luego dictan los institutos o universidades. Los institutos además pueden ofrecer otros cursos que tienen la desventaja de otorgar menor puntaje para su reconocimiento en la carrera. 
EI MEC desarrolla Campañas Nacionales de Capacitación para los docentes de todos los niveles, orientadas cada año a una temática diferente e involucra en su implementación a los Institutos de Formación Docente de todo el país.

En los países analizados la capacitación constituye un derecho de los docentes y a la vez una de las condiciones necesarias para sostener una educación de calidad, por lo que los estados intentan garantizar la igualdad en el acceso a través de la gratuidad. No obstante existe también una oferta privada, arancelada. La mayor dificultad en el acceso se observó en Paraguay donde, más allá de las Campañas Nacionales, la mayor parte de la oferta y actividades de formación continua son pagas y resultan insuficientes en cantidad, es decir que no cubren las demandas de los docentes y del sistema.

En síntesis, en los países con estructura federal convive una oferta organizada por diversos niveles de gobierno, centrales y locales y una multiplicidad de instituciones que desarrollan actividades de formación permanente (institutos, gremios, ministerios, universidades, municipios, etc.), mientras que en los países de estructura centralizada la capacitación de los docentes está a cargo directamente de los ministerios nacionales y los organismos que éstos designen. Esto puede acarrear dificultades en términos de la cobertura y disponibilidad de las acciones y programas para los docentes que residen en el interior de esos países.

\section{Modalidades, contenidos y enfoques del desarrollo profesional docente}

En todos los países del MERCOSUR se observa una variedad de formatos, modalidades y programas. Se destacan dos grandes grupos: los cursos de capacitación, actualización o formación continua y, por otra parte, las especializaciones, estudios o carreras de posgrado. Dentro de estos últimos se distinguen a su vez diferentes niveles de acuerdo a la duración de las carreras, exigencias y requisitos de ingreso: especializaciones o diplomaturas, seguido por las maestrías.

Los cursos en tanto formato masivo, tradicional y "de amplio espectro", siguen predominando. No obstante, junto a ellos se identifican nuevos dispositivos y formas alternativas centradas en el análisis y reflexión de las prácticas y en la implementación de proyectos en las escuelas. Estas propuestas de formación comienzan a coexistir como instancias o momentos (generalmente no presenciales) que se recortan y diferencian del trabajo más teórico centrado en saberes formales y actualización de contenidos didáctico-disciplinares.

Asimismo, se visualizan en la región actividades más informales y autónomas por su convocatoria y por la participación de los docentes como por ejemplo jornadas, congresos, ateneos o conferencias que empiezan a figurar como parte de las propuestas. Este tipo de acciones es particularmente visible en Brasil dado el protagonismo de las Universidades en la formación continua, aunque también se ha identificado en Argentina.

A diferencia de los demás países del MERCOSUR, Paraguay imparte fundamentalmente las denominadas "profesionalizaciones" destinadas a docentes en ejercicio con estudios secundarios y que no cuentan con las titulaciones requeridas de nivel superior para desempeñarse en el área o ciclo educativo correspondiente. Las profesionalizaciones son equivalentes y supletorias de la formación inicial ${ }^{11}$. En Brasil y en menor medida en Argentina también permanece parte de este tipo de oferta "supletoria"12.

Respecto de las modalidades, en los países analizados se observa un uso cada vez más generalizado de cursos semipresenciales o que combinan instancias presenciales y otras que no lo son, a través de la utilización de plataformas e-learning (o aprendizaje en línea). En Brasil y Uruguay la mayoría de la oferta relevada tiene al menos una parte de carga horaria no presencial. Es interesante destacar que este recurso ha renovado las estrategias y dispositivos de trabajo al enfatizar la participación y producción de los docentes en torno a proyectos, el intercambio de ideas y la socialización de experiencias. Asimismo ha permitido ampliar la cobertura, llegar a un mayor número de docentes. 
Por una parte, las áreas y temas de la formación continua en los países del MERCOSUR son muy amplios y heterogéneos. No obstante se encuentra mayor número de cursos, especializaciones y posgrados centrados en la actualización de contenidos de las disciplinas curriculares y sus estrategias de enseñanza. Por otra parte, se identifica en la región una preocupación por brindar herramientas y estrategias didácticas en relación con contextos escolares específicos de desempeño. Varios cursos y propuestas de formación permanente se refieren a temas y problemas contemporáneos, de actualidad educativa y social o que a veces se incluyen de manera transversal en los currículos (violencia escolar, derechos humanos y ciudadanía, medio ambiente, contextos de vulnerabilidad, desigualdad escolar, nuevas configuraciones familiares, la escuela en contextos de cambio, etc.). Las nuevas temáticas, tales como educación sexual y TIC tienen una presencia considerable.

En cuanto a los modelos y enfoques de la formación, se caracterizan por la heterogeneidad. Convive una modalidad de cursos más tradicionales centrados en la actualización de saberes, con otros que proponen la reflexión y transformación de las prácticas. A continuación se describen sucintamente las experiencias que recogen enfoques más actuales en los países del MERCOSUR, aunque conviven con otros más tradicionales o centrados sólo en la actualización de saberes disciplinares.

En Argentina se recupera la noción de formación centrada en la escuela, el programa de acompañamiento a noveles, que es una iniciativa importante en este nuevo marco. El desarrollo profesional de los docentes se propone utilizar una diversidad de modalidades, dispositivos y líneas de acción, sin olvidar las condiciones de trabajo y la necesidad de articular la formación con la carrera y con el puesto laboral. En Brasil algunas universidades desarrollan modalidades formativas menos ortodoxas tales como proyectos específicos de intervención, investigaciónacción e innovación en las escuelas donde los estudiantes realizan las residencias pedagógicas. Entre los principios de la "Política Nacional de Formación de profesionales de la Educación Básica" Brasil menciona una serie de fundamentos coherentes con las tendencias actuales en materia de desarrollo profesional docente, entre las que se destacan: la articulación teoría/ práctica en la formación docente, unión entre enseñanza, investigación y extensión; la escuela como espacio fundamental para la formación docente; la valorización de los distintos saberes y de la experiencia docente; la concepción de los docentes como agentes culturales.

Una concepción similar, dentro del mismo enfoque del desarrollo y aprendizaje docente, sostienen los Lineamientos de Formación Docente Continua de Argentina. Por su parte en Uruguay se destacan dos propuestas de carácter innovador. La primera son los "Módulos de Formación en Derechos Humanos", con una estructura abierta (conformada por diversos módulos) que aspira a convertirse en un trayecto a armarse según los intereses, posibilidades y trayectoria de cada docente. La segunda es el "Programa de Apoyo a la Escuela Pública Uruguaya" (PAEPU) destinado a la formación de docentes de las escuelas de contexto sociocultural crítico. Actualmente los cursos se han extendido a escuelas comunes, incluyen a los docentes y directores, alternan el trabajo presencial, la formación teórica con la elaboración y puesta en práctica de proyectos pedagógicos mediante estrategias de acompañamiento in situ a los docentes para su implementación. Se destaca además el componente de formación de formadores, la organización de equipos de trabajo y el desarrollo de materiales propios. 
En conjunto los países del MERCOSUR muestran que la formación docente continua es un tema de agenda pública que se caracteriza por su variedad, amplitud y dinamismo. La incorporación de nuevos temas y dispositivos de trabajo comienza a reflejarse en las propuestas. Dada la función que cumple la formación en servicio y las cambiantes necesidades de los sistemas educativos, sería esperable que estas tendencias se consolidaran y profundizaran generando a su vez una base común para el intercambio de experiencias y la movilidad de los docentes en la región.

\section{¿Es posible la convergencia entre los sistemas de formación docente del MERCOSUR?}

A partir de los propósitos iniciales del estudio, a continuación se plantean algunos ejes sobre los cuales el análisis realizado muestra que es posible avanzar en vistas a fortalecer, armonizar y articular la formación docente entre los países del MERCOSUR y, en el futuro, establecer marcos reguladores comunes.

En relación con la formación inicial se advierte una semejanza estructural en la duración de los estudios. La extensión de los planes es similar y, por lo tanto, constituye uno de los aspectos a considerar en el camino hacia la integración regional. Si bien una mayor duración de las carreras no necesariamente se vincula con una mejor preparación de los docentes, resulta conveniente asegurar condiciones básicas comunes para el conjunto de la educación superior, lo que permitiría además de fortalecer la formación específica, articularla con ofertas superiores de posgrado.

Otro criterio a considerar para definir políticas de integración regional de los planes de formación docente es la flexibilización curricular. ¿Hasta qué punto los planes de estudio de cada país habilitan para desarrollar espacios curriculares que aborden problemáticas regionales, o que, por ejemplo, incorporen otras lenguas? Asimismo cabe preguntarse si los contenidos seleccionados posibilitan formar docentes con capacidades, competencias o habilidades que les permitan abrir su horizonte cultural, contemplar otras realidades y escenarios para su quehacer, más allá de los nacionales. El análisis realizado mostró potencialidades en este aspecto al existir en los planes espacios de definición institucional o materias electivas en algunos países. Asimismo el componente de evaluación curricular permitiría introducir los cambios que se acuerden en la región. Otra opción podría consistir en reservar un pequeño porcentaje de horas del plan a contenidos de interés regional, más allá de los idiomas.

Un tercer criterio se refiere al perfil docente que aspira a formarse. Se infiere del estudio realizado que el conjunto de las capacidades y competencias propuestas en los planes de formación de los países del MERCOSUR son compatibles y complementarias. Más allá de la distinción de los términos utilizados (competencias o capacidades) en todos los casos se evidencia una preocupación por formar docentes que puedan actuar, tomar decisiones fundadas e indagar sobre sus prácticas educativas sentando las bases para la mejora continua de la enseñanza. Por lo tanto podría ser enriquecedor definir un perfil docente para la región que las abarque y que oriente la formación en base a las dimensiones de la profesión ya señaladas.

En relación con la formación continua tres son los aspectos que se destacan en la búsqueda de convergencias entre los países de la región. El primero se refiere a la flexibilidad de las temáticas y de los dispositivos. Este rasgo, presente con distintos énfasis en cada país, es un elemento central ya que permite orientar las actividades de capacitación en función de las cambiantes demandas del sistema y adaptarlas a los diversos contextos de desempeño, trayectorias y necesidades formativas de los sistemas educativos y de los docentes. En este sentido se destacan los esfuerzos de los países del MERCOSUR por ofrecer trayectos de formación continua de mayor alcance y duración que los cursos, seminarios y talleres aislados. 
Tales programas permitirían profundizar en determinadas problemáticas (educación rural, educación en contextos críticos); especializar los perfiles docentes en ciertas disciplinas y/o ciclos de la enseñanza (coordinadores de área curricular, alfabetización inicial, etc.) y formar para roles específicos (tutores, docentes orientadores de noveles, etc.) Dentro de este mismo aspecto también es necesario intensificar los dispositivos de formación que incorporan el análisis de las prácticas, la formulación de propuestas pedagógicas concretas y el seguimiento/apoyo a los docentes en las instituciones.

La segunda cuestión que podría ser parte de una estrategia de integración regional es la utilización de las plataformas e-learning y las modalidades a distancia, ya utilizadas por cada país para la formación continua de los docentes. Sin duda este rasgo puede ser capitalizado y aprovechado para diseñar plataformas de aprendizaje y desarrollo docente de carácter regional, basadas tanto en el intercambio de experiencias como en la formación de contenidos y generar propuestas de formación comunes para el MERCOSUR.

Por último, la formación continua, dada su reciente institucionalización y carácter dinámico, muchas veces ha quedado sujeta a lógicas de mercado, al margen de las regulaciones de los Estados. Sin embargo la definición de parámetros consensuados que permitan establecer criterios de pertinencia, calidad y evaluación de las ofertas podría elevar el nivel de las propuestas, resguardando además de posibles inequidades y de que los docentes más alejados de las grandes ciudades e instituciones queden a merced de programas de baja calidad y rigor académico, produciendo el efecto no deseado de la descualificación de su autonomía profesional.

\section{Bibliografía}

Alliaud, A. (2010). La formación docente. Estado de situación y desafíos a futuro. Voces del Fénix. Revista del Plan Fénix. Año 1, Núm. 3, 22-25. Disponible en: www.vocesenelfenix.com

Ávalos, B. (2007). Formación Docente Continua y factores asociados a la política educativa en América Latina y el Caribe. Informe preparado para el Diálogo Regional de Política, Banco Interamericano de Desarrollo.

Braslavsky, C. (1999). Re-haciendo escuelas. Hacia un nuevo paradigma en la educación latinoamericana. Buenos Aires: Santillana - Andrés Bello.

Barboza, L. y Nosiglia, C. (2011). La regulación de la Formación Docente: estudio comparado Argentina-Uruguay. IV Congreso Nacional / III Encuentro Internacional de Estudios Comparados en Educación: “¿Hacia dónde va la Educación en la Argentina y en América Latina? Construyendo una nueva agenda". Sociedad Argentina de Estudios Comparados en Educación.

Catti, B; Barretto, E.; Alonso André, M. (2011). Políticas docentes no Brasil. Um estado da arte. Brasília: Ministério da Educação de Brasil - UNESCO / Representação no Brasil.

Davini, M. C. (1995). La formación docente en cuestión: política y pedagogía. Buenos Aires: Paidós.

Davini, M. C. (2005). Estudio acerca de la calidad y cantidad de oferta de la formación docente, investigación y capacitación en la Argentina. Buenos Aires: Dirección Nacional de Gestión Curricular y Formación Docente, Ministerio de Educación.

Terigi, F. (2012). Los saberes docentes. Formación, elaboración en la experiencia e investigación. Documento Básico. Buenos Aires: Fundación Santillana.

Vaillant, D. (2005). Formación de docentes en América Latina. Re-inventando el modelo tradicional. Barcelona: Octaedro. 
Vezub, L. (2007). La formación y el desarrollo profesional docente frente a los nuevos desafíos de la escolaridad. Profesorado. Revista de Currículum y formación del Profesorado, №11 (1), Universidad de Granada. Disponible en: http://www.ugr.es/ recfpro/rev111ART2.pdf

Vezub, L. (2013). Hacia una pedagogía del desarrollo profesional docente. Modelos de formación continua y necesidades formativas de los profesores. Revista Páginas de Educación, Vol. 6, Núm. 1, 97-124. Montevideo: Universidad Católica de Uruguay http://paginasdeeducacion.ucu. edu.uy/inicio/item/43-pags_edu6.html

\section{Documentos}

\section{Argentina}

Ley de Educación Nacional N²26.206, año 2006.

Resolución del Consejo Federal de Educación N² 24/07 y ANEXO I: "Lineamientos Curriculares Nacionales para la Formación Docente Inicial".

Resolución del Consejo Federal de Educación № 30/07 - ANEXO II: “Lineamientos Nacionales para la Formación Docente Continua y el Desarrollo Profesional”

Resolución del Consejo Federal de Educación N73/08 - ANEXO: "Recomendaciones para la adecuación de ofertas y títulos de Formación Docente Inicial a la Resolución CFE N²4/07".

Resolución del Ministerio de Educación N²170/08: "Procedimiento para otorgar validez nacional a títulos y certificados correspondientes a estudios de formación docente de todos los niveles y modalidades del sistema formador".

Instituto Nacional de Formación Docente, Ministerio de Educación, 2010. "Núcleos temáticos prioritarios - Proyecto de mejora para la formación inicial de profesores para el nivel secundario".

\section{Brasil}

Diretrizes Curriculares Nacionais para a Formação de Professores da Educação Básica, em nível superior, curso de Licenciatura, de Graduação plena. Resoluçao CNE/CP 1/2002; Pareceres CNE/CP 9/2001 e 27/2001.

Diretrizes Curriculares Nacionais do curso de pedagogia. Resolução: CNE/CP 1/2006, Pareceres CNE/CP 5/2005 e 3/2006.

A duração e a carga horária dos cursos de licenciatura, de graduação plena, de formação de professores da Educação Básica em nível superior. Resolução: CNE/CP 2/2002.

Ley Federal de Educación de Brasil № 9.394, año 1996 (Ley de Directrices y Bases de la Educación Nacional).

Ley $\mathrm{N}^{\circ} 12.056$, año 2009

Ley $\mathrm{N}^{\circ} 12.796$, año 2013

Portaria MEC No 1.179. Sistema Nacional de Certificação e Formação Continuada de Professores - Rede Nacional de Formação Continuada de Professores. Universidade Aberta do Brasil (UAB).

\section{Paraguay}

Ley General de Educación No 1264, año 1998

Diseño Curricular de la Formación Docente Inicial para la Educación Inicial. Ministerio de Educación y Cultura. 2006-2008

Diseño Curricular de la Formación Docente Inicial para la Educación Escolar Básica $1^{\circ}$ y $2^{\circ}$ ciclos. 2013. Ministerio de Educación y Cultura. 
La formación inicial y continua de los docentes en los países del MERCOSUR ...

Marco Curricular para la Formación Docente Continua Inicial de la Educación Media. 2010.

\section{Uruguay}

Acta No. 63 Res. No. 67, ANEP, 18 de octubre de 2007. Sistema Único Nacional de Formación Docente 2008 - Documento Final.

Acta N³1 Res. 22 de ANEP, del 19 de mayo de 2009: aprueba Reglamento para los Diplomas, Especializaciones y las Maestrías dependientes del Área de Perfeccionamiento y Estudios Superiores.

Acta Extraordinaria N5, 24 de junio 2010 - Creación del Consejo de Formación en Educación Convenio entre la Universidad de la República (UDELAR) y la Administración Nacional de la Educación Pública (ANEP) 29/07/2009

Ley General de Educación № 18.437, 12 de diciembre de 2008

Protocolo para la homologación de cursos de actualización por parte del Consejo de Formación en Educación. Instituto de Perfeccionamiento y Estudios Superiores "Prof. Juan E. Pivel Devoto". Consejo de Formación en Educación - ANEP. 2013.

\section{Notas}

${ }^{1}$ El Programa de Apoyo al Sector Educativo del Mercosur (PASEM) constituye una actividad conjunta del MERCOSUR con la Unión Europea que busca promover el rol central de la educación en los procesos de integración de la región. Su objetivo general es contribuir a la mejora de la calidad educativa a través del fortalecimiento de la formación profesional docente en Argentina, Brasil, Paraguay y Uruguay.

${ }^{2}$ El estudio fue coordinado por la Oficina OEI en Buenos Aires y se llevó a cabo por parte de un equipo conformado por Andrea Alliaud (coordinadora general) y Paulo Henrique Arcas, Silvina Feeney y Lea Vezub en carácter de expertos nacionales. Contó además con la asistencia técnica de Carolina Lifchitz y Marina Elberger. Asimismo participaron Miquel Martínez y Enrique Prats en la sistematización de la información sobre los sistemas de formación docente en la Unión Europea.

${ }^{3}$ Entendemos por dispositivos de formación al conjunto de experiencias formativas consideradas especialmente potentes para que los docentes puedan desarrollar el juicio práctico ineludible a su quehacer docente. Formas de formar que vinculan la teoría y la práctica, el pensamiento y la acción.

${ }^{4}$ La Universidad privada de Montevideo ha sido habilitada por el CODICEN desde 2008 para brindar planes de formación inicial de magisterio y de profesorado (en cinco especialidades). La Universidad Católica ofrece como nueva carrera el plan de maestros de primaria en sus tres sedes.

${ }^{5}$ Cabe resaltar que las regulaciones establecen la carga horaria mínima. A partir de allí, en los países descentralizados se definen planes que pueden tener más horas de duración. En estos mismos países los profesorados que imparten las universidades duran entre 4 y 5 años como mínimo.

${ }^{6}$ Se entiende por flexibilidad curricular los grados de libertad que el plan de estudios permite para definir otras opciones no contempladas, no explicitadas, en el marco común general, tanto a nivel de las administraciones educativas locales, como de las instituciones y de los actores del sistema (estudiantes y profesores).

${ }^{7}$ Las dimensiones especificadas han sido revisadas y reelaboradas a partir de las aportadas en el trabajo de Braslavsky (1999). 
${ }^{8}$ El proceso de adecuación que están protagonizando las universidades argentinas supone, entre otras cuestiones, un tratamiento diferencial de las prácticas respecto de los planes existentes, a fin de contemplar su inicio desde los primeros años de la carrera mediante actividades que permitan analizar y reconstruir situaciones propias del quehacer docente.

${ }^{9} \mathrm{~A}$ este respecto, en Argentina se destaca la importancia de lograr la articulación de los conocimientos prácticos y de los brindados por los otros campos curriculares; en Paraguay se plantea "confrontar de manera sistemática su formación teórica con las dificultades concretas de las situaciones de enseñanza, en las diferentes modalidades y contextos educativos".

${ }^{10} \mathrm{El}$ objetivo y características de las profesionalizaciones de Paraguay se describen en el apartado siguiente.

${ }^{11}$ Las profesionalizaciones vigentes en Paraguay al momento del estudio eran: en Educación Inicial; en Escuelas Indígenas; en las disciplinas de Ciencias Básicas, Educación Física, Matemática y Ciencias Sociales, Lengua y Literatura Castellana y Guaraní, Educación Artística para Educación Media y Tercer Ciclo; en Lengua extranjera (inglés) para Educación Media, y en Trabajo y tecnología para Tercer Ciclo.

${ }^{12}$ Los datos muestran que todavía hay un porcentaje de docentes en las escuelas públicas sin formación superior: en la Educación Infantil un 36,4\%; en los años iniciales de la Enseñanza Fundamental un 25\% y en años finales 9,6\%; en la Enseñanza Media la proporción se reduce al 4,6\% de profesores sin título superior. Hasta el año 2012, de acuerdo con las informaciones presentes en la página del MEC, el PARFOR, creado en 2009, había capacitado a 54.000 profesores de educación básica en 397 municipios del país. 